Gesnerus 66 (2009) 40-66

\title{
Décrire et convaincre: rhétoriques visuelles de la cinématographie en médecine
}

Francesco Panese

\section{Summary}

The tools of visualisation occupy a central place in medicine. Far from being simple accessories of glance, they literally constitute objects of medicine. Such empirical acknowledgement and epistemological position open a vast field of investigation: visual technologies of medical knowledge. This article studies the development and transformation of medical objects which have permitted to assess the role of temporality in the epistemology of medicine. It firstly examines the general problem of the relationships between cinema, animated image and medicine and secondly, the contribution of the German doctor Martin Weiser to medical cinematography as a method. Finally, a typology is sketched out organising the variety of the visual technology of movement under the perspective of the development of specific visual techniques in medicine.

Keywords: visual technologies; medical epistemology; Martin Weiser

\section{Résumé}

Les outils de visualisation occupent une place centrale en médecine. Loin d'être de simples accessoires du regard, ils constituent littéralement des objets du savoir médical. Ce constat empirique et cette posture épistémologique ouvrent un vaste terrain d'investigation: les technologies visuelles de la connaissance médicale. Cet article retrace le développement des techniques visant à saisir les mouvements et les transformations des objets en médecine, permettant ainsi d'asseoir la place de la temporalité dans son épis-

Francesco Panese, Institut universitaire d'histoire de la médecine et de la santé publique, CHUV et Faculté de biologie et de médecine, 1, chemin des Falaises, CH-1005 Lausanne, et Faculté des sciences sociales et politiques, Université de Lausanne (Francesco.Panese@unil.ch). 
témologie. Après une problématisation générale des relations entre cinéma, image animée et médecine, on aborde la contribution du médecin Martin Weiser, qui participa à l'instauration de la cinématographie en dressant en 1919 le vaste inventaire de ses usages médicaux. On esquisse ensuite une typologie apte à organiser la variété de ces technologies visuelles du mouvement sous l'angle du développement de rhétoriques visuelles médicales spécifiques visant simultanément à décrire et à convaincre grâce à des dispositifs permettant d'animer des traces, de séquencer des transformations ou d'orienter des comportements.

\section{Introduction}

Les relations entre cinéma ${ }^{1}$ et médecine composent un large territoire d'enquête et un corpus très diversifié, des travaux d'Etienne-Jules Marey à la série télévisée à succès Grey's Anatomy. Ces deux bornes au rapprochement de prime abord insolite indiquent une typologie possible de la grande quantité et de la variété des films relatifs d'une manière ou d'une autre à la médecine. On peut y distinguer sommairement trois grandes catégories, qui correspondent d'ailleurs à une typologie classique du film en général. Tout d'abord, celle qui regroupe les mises en fiction de la médecine, catégorie sans doute la plus étudiée ${ }^{2}$. Bien qu'elles ne fassent pas l'objet de la présente étude, signalons que ces fictions cinématographiques ou télévisuelles composent aujourd'hui un genre à part entière qui tend d'ailleurs à proliférer sur les petits écrans. Dans la perspective d'une sociologie de la culture, elles sont loin d'être anecdotiques. Elles constituent sans doute un important répertoire de représentations qui participent, à des degrés divers, à façonner les représentations sociales de la pratique médicale, de la maladie, de la santé, de la normalité, sous la forme de clichés, de stéréotypes, mais aussi, il faut le souligner, de connaissances et de compétences qui peuvent être mobilisées

1 Nous regroupons pour l'heure ici sous la notion de «cinéma» la grande diversité des modalités des «images en mouvement» utilisée par, ou faisant référence à, la médecine. Nous préférerons souvent l'ancienne notion générique de «cinématographie» présente dans les sources pour éviter de dénoter de manière exclusive le cinéma de fiction projeté en salle. Le «film» renverra de manière plus spécifique aux documents et l'«image animée» à un support unique sur lequel vient s'inscrire un mouvement ou une séquence. Nous reviendrons sur ces distinctions. Une part des sources que nous analysons ici a été identifiée dans le cadre du séminaire «Science, médecine et société» que nous avons tenu à l'Université de Lausanne en 2007 sur le thème «Médecine, science, image et cinéma» avec mes collègues Cynthia Kraus et Vincent Pidoux auxquels va ma gratitude ainsi qu'à Vincent Barras, lecteur patient de cette étude en train de se faire.

2 Cf. Frayling 2005; Wider 1990; Harper/Moor 2005. 
par le public lorsque les individus qui le composent sont confrontés à la médecine ${ }^{3}$. La seconde catégorie concerne le large éventail du film dans la communication médicale, du cinéma sanitaire à la vulgarisation ${ }^{4}$, sans qu'il soit toujours facile - ou pertinent - d'y distinguer la part du «documentaire» et de la «fiction». La troisième catégorie enfin regroupe ce qui fut sans doute les premiers fruits de l'alliance entre l'image animée et la médecine, soit des documents produits par des technologies ${ }^{5}$ visuelles de la connaissance qui permettent, dans des contextes spécifiques, de produire des descriptions intelligibles et pertinentes d'objets médicaux dont l'existence iconographique est d'autant plus évidente qu'elle semble apparemment ne pas reposer sur un «récit».

Cette typologie sommaire témoigne de la diversité des fonctions relatives à l'usage du film et des images animées en médecine: divertir, enseigner, faire croire, faire voir, observer ou encore, de manière plus générale, décrire et convaincre, soit autant de fonctions qui participent de la rhétorique visuelle médicale dont ces outils ont été très tôt de nouveaux moyens. Nous en analyserons ici quelques facettes en partant du précieux bilan sur les rapports entre médecine et cinématographie établi en 1919 déjà par Martin Weiser. En adoptant délibérément à sa suite une définition à la fois minimale et large de la cinématographie comme «écriture du mouvement», nous poursuivrons l'enquête en nous attachant à décrire des dispositifs techniques et épistémiques qui permettent de construire la visualité d'actions ou de transformations temporelles de sujets, d'objets ou de phénomènes dans le contexte médical ou apparenté. Nous tenterons en particulier de mettre en évidence la manière dont le film a souvent pris le relais de l'image fixe pour capturer ces actions ou ces transformations. Nous illustrerons enfin la persistance de la rhétorique visuelle de la «preuve» en médecine dans le cinéma sanitaire comme outil épistémique et social de l'orientation des comportements de santé.

3 Ce fut le cas notamment avec la série Urgences qui donna lieu à de nombreux travaux. Cf. O’Connor 1998; Peneff 1998; Chalvon-Demersay 1999.

4 Sur la vulgarisation médicale télévisée, cf. Zappalà 1997.

5 Nous entendons cette notion au sens que lui donnent Steven Shapin et Simon Schaffer, qui ont décrit l'émergence des sciences expérimentales modernes comme la mise en œuvre de trois technologies en tant que «knowledge-producing tools»: «matérielle» (les dispositifs expérimentaux), «littéraire» (l'écriture scientifique et ses moyens de diffusion) et «sociale» (la constitution d'une communauté de pairs) qui constituent autant d'outils de production des connaissances, s'articulant les uns aux autres. Cf. Shapin 1995; Shapin/Schaffer 1993. Nous reviendrons plus loin sur la définition de la notion d'«image animée». 


\section{Martin Weiser: la cinématographie comme méthode}

Au sortir de la Grande Guerre, alors que le cinéma de fiction se mue progressivement en industrie ${ }^{6}$, Martin Weiser, «médecin d'état-major et conseiller pour la radiologie auprès du XII ${ }^{\text {ème }}$ Corps d'armée de Dresde» fait le point sur les nombreux apports des techniques cinématographiques aux sciences médicales. Dans la préface de sa «Medizinische Kinematographie» ${ }^{7}$, un ouvrage de cent cinquante pages truffé de références, il indique que la cinématographie est un outil d'apprentissage et de recherche («Lehr- und Forschungsmittel») sur le point de se généraliser en médecine:

Vielleicht ist die Zeit nicht mehr fern, wo die Kinematographie ein sicheres Hilfsmittel eines jeden Universitätsinstitutes sein wird. ${ }^{8}$

Ce constat prophétique témoigne sans doute de l'attirance de la médecine de l'époque pour la «nouveauté», dans une phase importante de sa modernisation et de sa technicisation. Sur le plan épistémologique, la lecture de Weiser donne surtout l'impression que cette nouvelle technologie visuelle est apparue dès son origine comme particulièrement adéquate aux développements médicaux, comme si elle permettait de mener plus avant ce que la médecine faisait déjà par d'autres moyens ${ }^{9}$. Cette adéquation surprenante découle sans doute de l'une des fonctions heuristiques du film: être un outil de description des transformations temporelles des objets.

En 1919, cette fonction élémentaire nécessite (encore) une démonstration détaillée. Suivant la voie ouverte par Eadweard Muybridge et surtout par Marey dont il explicite les procédés, Weiser déploie dans son ouvrage le large panorama des usages de la «cinématographie» en médecine. Il nous indique ainsi une clé de lecture qui peut, aujourd'hui encore, nous servir de guide: la prolifération de ses usages participe d'une construction technique, épistémologique et sociale des phénomènes médicaux que les films prennent pour objets; corollairement, les modes d'existence des objets filmiques de la mé-

6 En 1919, année de publication de l'ouvrage de Weiser, Charles Chaplin, Douglas Fairbanks, Mary Pickford et D. W. Griffith fondent United Artists et les premières superproductions remplissent les salles qui se multiplient.

7 Martin Weiser 1919. Malgré sa taille modeste, l'ouvrage de Weiser vise à documenter et à référencer l'intégralité de la production cinématographique dans le domaine de la médecine, de Marey à 1918. Il constitue de ce point de vue un catalogue raisonné particulièrement précieux de ses multiples usages.

8 Weiser 1919, 1.

9 Cette situation rappelle le cas des Rayons X qui pénètreront et façonneront la pratique médicale avec une rapidité impressionnante. En effet, dès la publication de 1895 par Röntgen, la médecine s'empare de la découverte et l'adapte pour poursuivre dans le régime du visuel les techniques «médiates» de diagnostic interne jusqu'alors limitées à l'exercice de l'ouïe et du toucher, via le stéthoscope, la percussion et la palpation, pour cerner les conformations internes des corps. Cf. Pasveer 1993; 1995. 
decine sont inséparables des modes de narration spécifiques au cinématographe. Pour illustrer cette perspective, il vaut la peine de rapporter brièvement la démonstration du médecin cinéaste. Son traité s'ouvre par l'exposé technique de la «Normalkinematographie». Description des caméras disponibles, techniques de prises de vue, traitement de la pellicule, etc.: l'exposé est celui d'un nouvel outil d'investigation à même de fixer les phénomènes temporels pertinents pour la médecine. Comme cela a été le cas des usages scientifiques de la photographie, considérée comme un moyen privilégié de l'objectivité ${ }^{10}$, il est intéressant de constater qu'aucun domaine médical ne semble échapper à l'opportunité de tirer profit des usages et des potentialités diverses et multiples du film et de l'image animée. Nombreuses sont en effet les situations que les médecins imaginaient d'améliorer grâce à ces différentes techniques. La «cinématographie normale» (Normalkinematographie) tout d'abord, qui consiste à capturer le visible en action tel qu'il s'offre spontanément au regard, apparaît comme particulièrement adaptée à la documentation et à la constitution d'une sorte de patrimoine cinématographique en «médecine générale» (Allgemeine medizinische Kinematographie). Weiser appelle ainsi de ses voux la multiplication des Meister Filme qui, comme leur nom l'indique, permettent de léguer à la postérité les gestes des «maîtres». Mais il insiste surtout sur les potentialités de cet outil pour fixer, conserver et montrer des techniques médicales (Arbeitsmethoden) dans le cadre de la formation clinique (Klinischer Unterricht) ou de la formation continue dispensée par les Sociétés médicales (Fortbildungskurse), une vocation pédagogique promise à un immense développement dans les auditoires parfois encombrés d'étudiants ou de spectateurs. Ce «précieux outil de recherche scientifique» ${ }^{11}$ est en outre un outil de conservation qui permet aux objets variés de la médecine de rejoindre des archives scientifiques spécifiques (Wissenschaftliche Filmarchive) à l'instar de celui de la KaiserinFriedrich-Haus de Berlin.

En bon scientifique, Weiser connaît les limites des outils qui ne font que reproduire le visible, presque sans transformation. Si la Normalkinematographie réplique le réel, les «cinématographies médicales spéciales» (spezielle medizinische Kinematographien) permettent d'agir sur le visible et/ou sur le temps, afin de capturer des situations ou des phénomènes qui, sans l'intervention de technologies visuelles spécifiques, resteraient invisibles et illisibles à l'œil de l'observateur. Un premier dispositif consiste à coupler la Normalkinematographie à d'autres outils, tels que le microscope (Mikrokinemato-

10 Sur cette quête d'«objectivité mécanique» par les moyens photographiques, poursuivie ici par les moyens cinématographiques, cf. Daston/Galison 2007, en particulier chap. 3; 1992. 11 Weiser 1919, 90. 
graphie) ou l'installation radiologique (Röntgen-Kinematographie) ${ }^{12}$, afin de faire accéder à la visualité des corps en mouvement invisibles du fait de leur petitesse ou de leur opacité. Un second, complémentaire au premier, inspiré par la chronophotographie ${ }^{13}$ (Hochfrequenzkinematographie ${ }^{14}$ ), consiste quant à lui à fixer et à rendre lisibles des mouvements trop rapides ou trop lents pour être perçus.

De ces deux dispositifs élémentaires découle l'inventaire impressionnant des applications de la cinématographie aux diverses spécialités médicales. Dans chaque cas, elles permettent de donner une visualité nouvelle à des phénomènes «classiques» dont voici quelques exemples inventoriés par Weiser: mouvements des corps et de ses parties, le cœur en particulier, pour l'anatomie et la physiologie; développement cellulaire en embryologie et en histologie; accidents électriques en «électropathologie»; les techniques de production de substances et leur effets en pharmacologie; les «Tuberculosefilme», l'électro-, l'hydro- ou l'héliothérapie comme les cures de la tuberculose infantile, d'Auguste Rollier à Leysin ${ }^{15}$ en médecine interne; les opérations ou les prouesses comme celle du Dr Doyen en chirurgie ${ }^{16}$; les Kinematogramme d'accouchements en gynécologie; la description du nystagmus (mouvement d'oscillation involontaire et saccadée du globe oculaire) ou le maniement de divers outils adaptés aux aveugles en ophtalmologie; les crises ou les comportements des malades en neurologie et en psychiatrie; la chronophotographie de la parole en laryngologie et en otologie; les pratiques d'hygiène et les équipements en médecine militaire; et enfin, parmi tant d'autres encore, les nombreux films, documentaires ou de fiction, relatifs à

12 Notons ici le diagnostic une fois encore très perspicace de Weiser sur cette technique dont il fixe les premiers essais en 1909: «Es ist heute überhaupt kaum mehr die Rede von der Röntgen-Kinematographie. Sie liegt wie eine abgeschlossene Episode historisch geworden hinter uns. Ob es dabei bleiben wird? Es ist möglich. Versagt hat das Verfahren nicht. Das kann man nicht behaupten, denn es sind mit ihm eine Reihe wertvoller Kenntnisse zutage gefördert worden.» (Weiser 1919, 69) Comme nous le verrons, il ne sera pas contredit.

13 On reconnaît l'héritage de Marey et de son école, dont Weiser cite presque tous les travaux, à côté de ceux de la tradition allemande qui, on le sait, inspirera l'auteur de La méthode graphique dans les sciences expérimentales et particulièrement en physiologie et en médecine (Paris 1878).

14 Weiser commente le dispositif de Cranz et Glatzel capable de réaliser des prises de vue à 100000 images par seconde, en balistique et en «Funken-Kinematographie» qui consistait à saisir des phénomènes ondulatoires à haute fréquence.

15 Weiser mentionne «Die Schule an der Sonne» du Dr Rollier (date inconnue). Signalons ici la fortune cinématographique internationale de Rollier dont témoignent notamment «Klinieken van dr. Rollier te Leysin» de Dick Laan (1926), suivi l'année suivante de «Zonnekuren in de klinieken van Dr Rollier te Leysin» (1927), deux films catalogués dans la «Nederlandse Film Database» (http://nfdb.akris.nl).

16 Weiser a sans doute vu le fameux film du 9 février 1902 du Dr Doyen séparant les deux sœurs siamoises, vedettes du cirque Barnum, Doodica et Radica Neik, sous la caméra de Clément Maurice. Cf. Lefebvre 1994; 2002b; 2004. 
l'hygiène en général, des travaux d'assainissement des grandes villes aux maladies vénériennes.

\section{Pour une épistémologie historique des dispositifs visuels en médecine}

Les technologies visuelles décrites par Weiser, comme toutes celles qui accompagneront les développements de la médecine au cours du siècle, s'inscrivent dans le cadre d'une épistémologie médicale qui pose la maîtrise scientifique et expérimentale des transformations temporelles de ses objets au cœur de ses méthodes ${ }^{17}$. Dans ce contexte, les dispositifs de visualisation du mouvement remplissent différentes fonctions concomitantes: cognitives, en fixant par l'image ce qui sans elle échapperait à la perception; épistémiques, en conditionnant l'analyse des mouvements ainsi fixés; épistémologiques, en rapportant cette connaissance à un système théorique plus général que ces technologies contribuent en retour à façonner. Sur le plan heuristique, ces dispositifs produisent, sous des formes diverses, des inscriptions spatialisées et temporalisées de déplacements, de mouvements, d'événements ou de métamorphoses.

Comme nous le savons, la recherche de dispositifs visuels permettant de remplir ces différentes fonctions n'a pas attendu l'outil cinématographique. En effet, la figuration du mouvement, au sens général du terme, s'est largement développée, et depuis longtemps ${ }^{18}$, dans le cadre de ce qu'il est convenu d'appeler les images animées ${ }^{19}$, soit des inscriptions de traces, de formes ou de situations, figuratives ou indiciaires, sur des supports uniques ou sériels, structurées en flux ou en séquences, ordonnées dans l'espace temporellement structuré de la figure. Nonobstant la diversité technique et iconographique qui découle de cette combinatoire, toutes ces images consistent à articuler un lexique visuel et un lexique temporel, comme le fera le film: «Der Kinematograph», rappelle pertinemment Weiser, «beherrscht Zeit und Raum» ${ }^{20}$. Cette esquisse de formalisation permet d'identifier et de distinguer les opérations à la fois techniques et épistémiques que permettent les dispositifs visuels des images animées en médecine. Nous nous arrêterons ici sur quatre

17 Pour une approche générale, cf. Sturken/Cartwright 2001, en particulier le chap. 8, «Scientific looking, looking at science», 279-314.

18 Pour s'en convaincre, cf. l'excellente étude de Pierantoni 1986. L'auteur montre de manière convaincante que le défi de représenter le mouvement sur une image fixe est déjà lisible dans certaines représentations rupestres.

19 Nous reprenons le terme utilisé dans les études mareysiennes (Société d'études sur Marey et l'image animée SEMIA). Il désigne aussi les dispositifs qui permettent de visionner plusieurs images en une seule. Cf. Timby 2001.

20 Weiser 1919, 137. 
d'entre elles: animer des traces, séquencer des transformations, explorer l'intérieur des corps et orienter des comportements. Dans chaque cas, nous illustrerons la manière dont le film a prolongé ou complété la rhétorique visuelle des images animées par de nouveaux moyens.

\section{Animer des traces}

Comme l'ont montré de nombreux travaux ${ }^{21}$, c'est sans doute dans le contexte de la physiologie de la fin du XIX ${ }^{\mathrm{e}}$ siècle, en particulier dans les travaux initiés par Etienne-Jules Marey, que l'on peut situer l'affirmation dans le champ médical, et dans ceux qui lui sont apparentés, d'une écriture et d'une lecture des corps par des instruments, inspirées par une approche physicienne et expérimentaliste de la mesure du mouvement ${ }^{22}$. Du sphygmographe à la chronophotographie, ces technologies consistent à inscrire le mouvement sous la forme visuelle d'une géométrie dynamique, par sa décomposition séquentielle. Leur principe consiste à rassembler l'espace et le temps en des inscriptions manipulables, mesurables et analysables. La raréfaction nécessaire de l'information toujours trop abondante provenant de la perception du réel conduit à abstraire, à des degrés divers, la figuration des corps en mouvement, permettant ainsi des «jugements perceptifs ${ }^{23}$ clairs et communicables. On le sait, la chronophotographie inventée par Marey constitue l'exemple par excellence de ce principe général: le corps réduit figurativement à une géométrie qui en simplifie la structure s'imprime en une forme d'emblée diagrammatique et mesurable sur la plaque photographique.

Outre l'ingéniosité du dispositif chronophotographique maintes fois commenté, il est utile de rappeler le paradigme plus large dans lequel il s'inscrit. Comme Marey l'avait bien établi ${ }^{24}$, cette «méthode graphique» consiste à inscrire le déploiement temporel de toute activité physiologique susceptible d'être transformée techniquement en tracé, comme c'est le cas dans les multiples techniques de monitorage qui ressortissent également du registre de l'image animée. Du point de vue d'une épistémologie historique, nous aimerions souligner le fait que ces dispositifs ne servent pas sim-

21 Cf. notamment Dagognet 1987; Braun 1994.

22 Cette approche est très bien illustrée par l'étude princeps que Galilée consacra aux taches solaires et à l'occasion de laquelle il mit au point une technologie visuelle permettant de séquencer et de suivre leur mouvement sous la forme de ce que l'on doit bien appeler un «flip book». Pour s'en convaincre, cf. Galilei 1613 auquel nous avons consacré une étude: Panese 1999.

23 Latour 1985.

24 Nous renvoyons bien sûr à Marey 1878. 


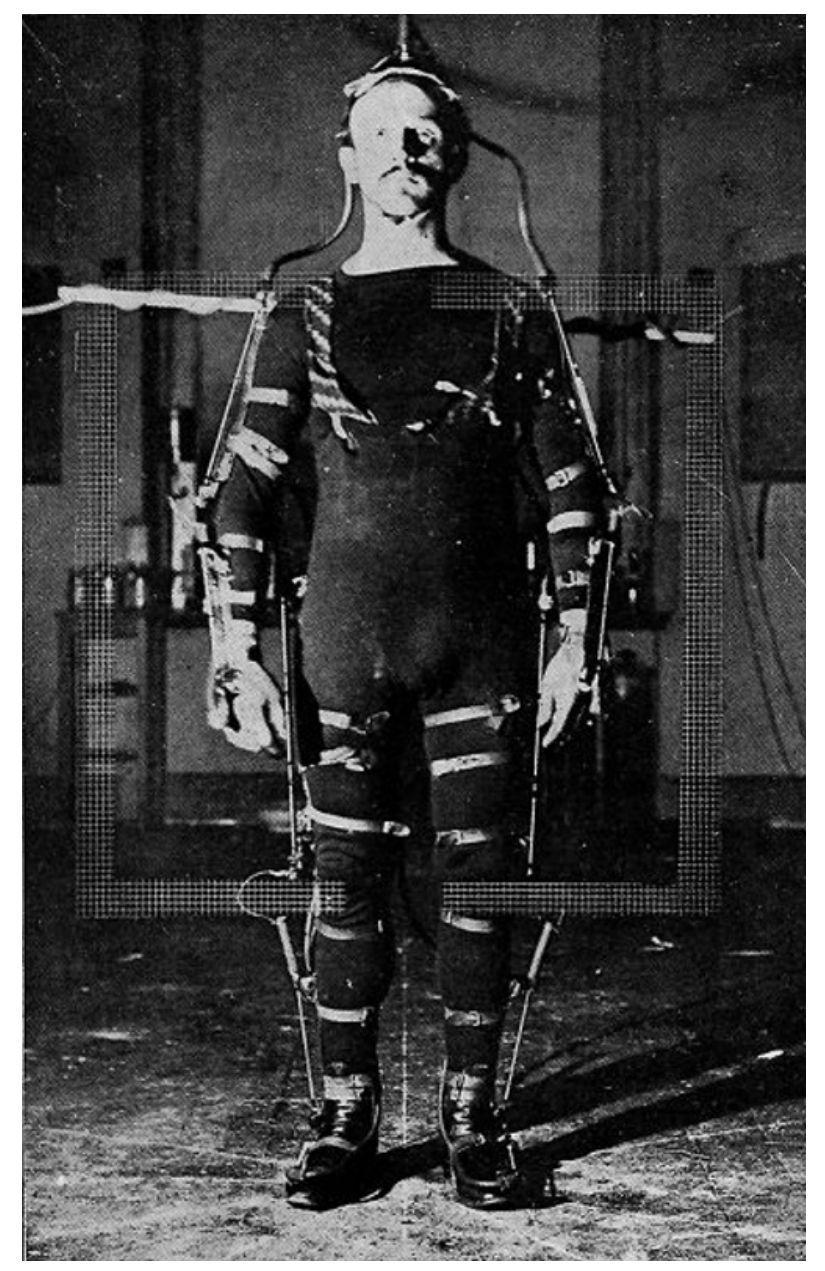

Fig. 1. «Das Versuchsindividuum in voller Ausrüstung», aus: W. Braune und O. Fischer, Der Gang des Menschen, dans: Weiser 1919.

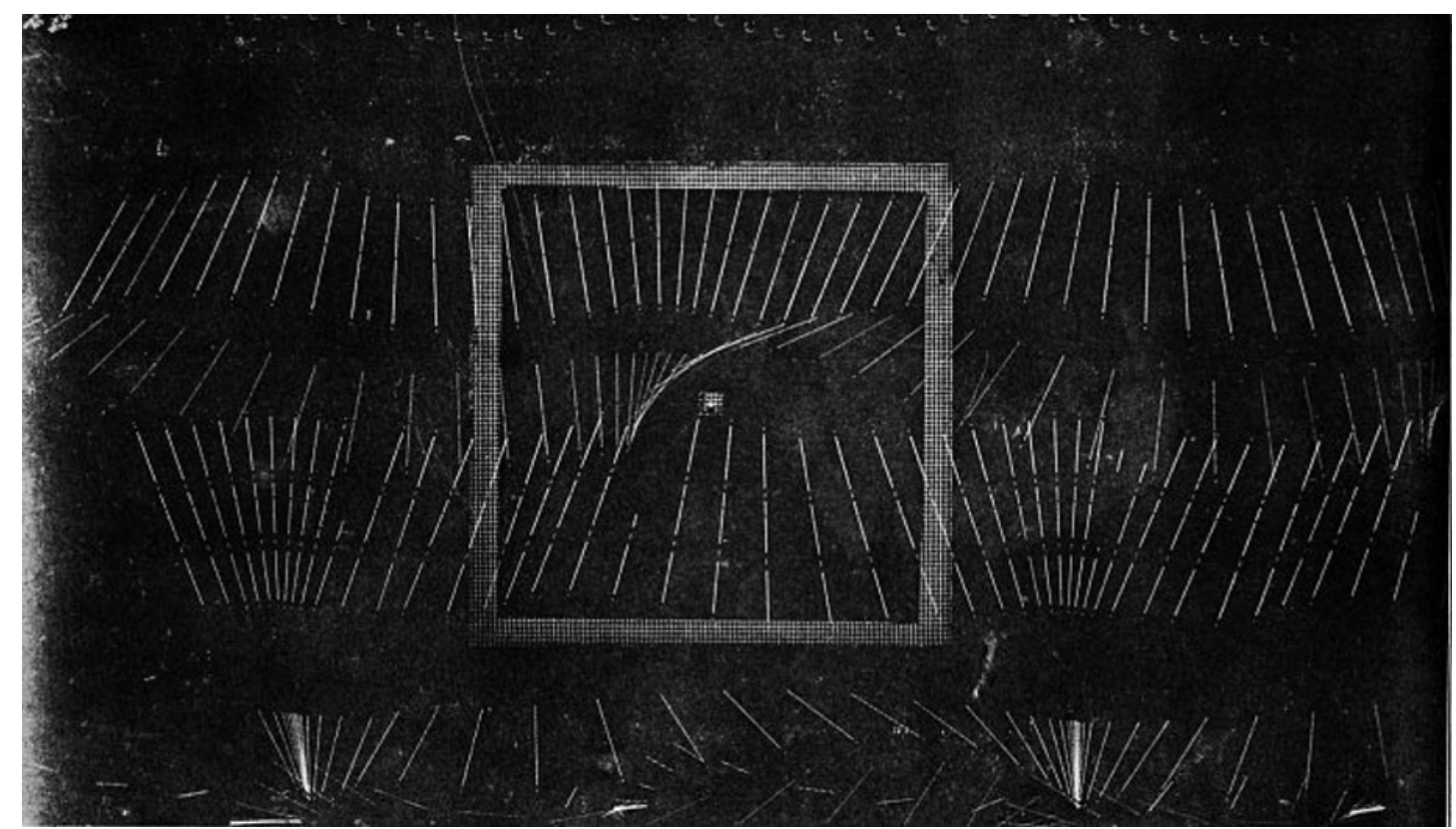

Fig. 2. «Eine chronophotographische Aufnahme von W. Braune und O. Fischer», dans: Weiser 1919. 
plement d'enregistreurs, mais qu'ils façonnent littéralement les objets de savoir qui ne pourraient exister sans eux ${ }^{25}$. Reconnaître cette dimension formative conduit à reconsidérer le rôle de ce genre d'inscriptions, souvent modestes, dans l'histoire des connaissances. Nous aimerions le suggérer par deux exemples contrastés. Le premier concerne la construction scientifique $\mathrm{du}$ «poumon physiologique» analysé par Vincent Barras ${ }^{26}$. Dans la vague d'études consacrées aux effets physiologiques du climat sur l'homme, et de l'altitude en particulier, on constate que les dispositifs enregistreurs construisent littéralement la lisibilité et l'intelligibilité des corps contraints par des conditions environnementales. Adolf Loewy présente ainsi un ingénieux et complexe dispositif portable d'enregistrement de la respiration qui permet de reconstruire par des tracés corrélés la temporalité physiologique du corps en ascension ${ }^{27}$.
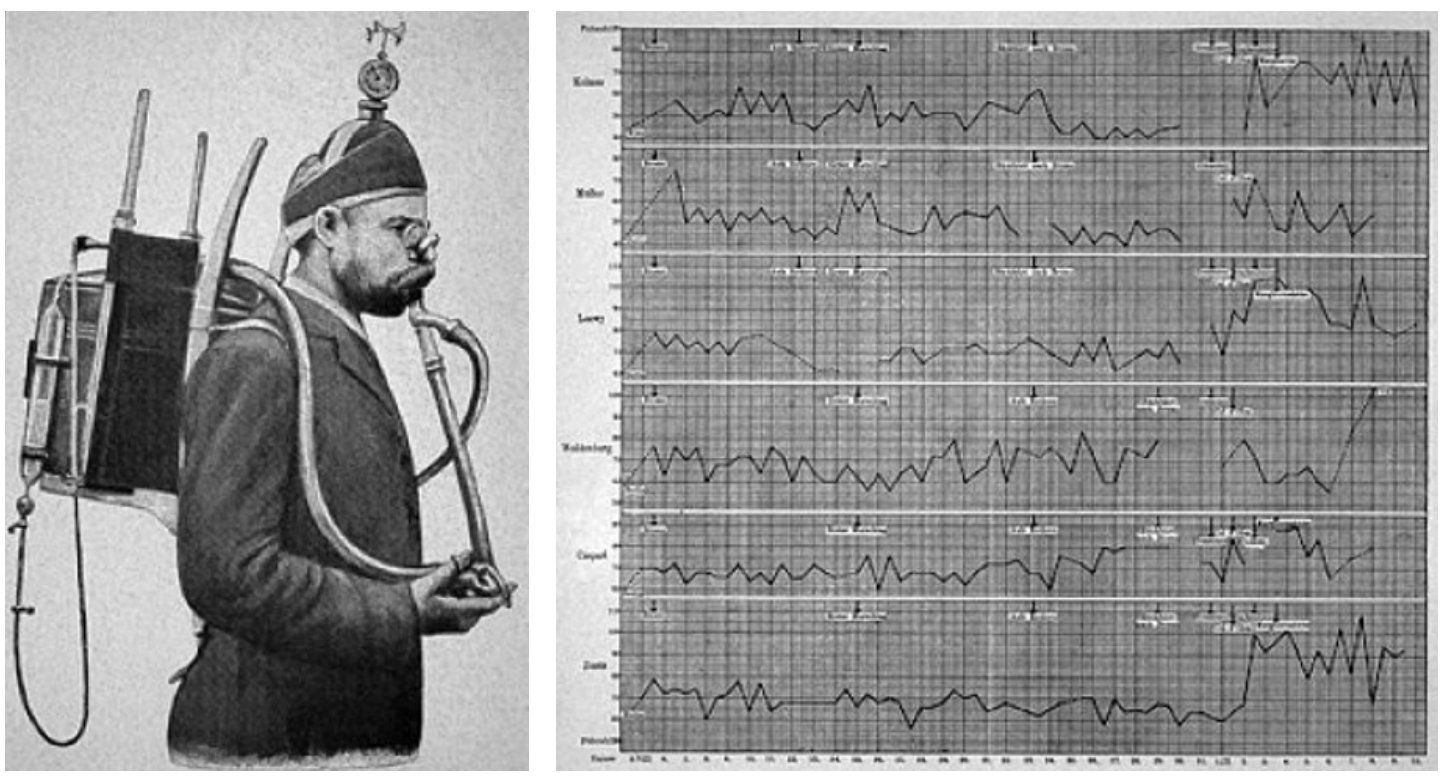

Fig. 3 et 4. Dispositif d'enregistrement de la respiration et tracé respiratoire, dans: Adolf Loewy, Physiologie des Höhenklimas (Berlin 1932).

Dans le fil épistémologique de l'anatomo-clinique qui fonde la lisibilité de l'espace des corps, ces dispositifs marquent la conquête technicienne de la temporalité en médecine, participant ainsi de l'affirmation en son sein d'une forme renouvelée d'expérimentalisme basé sur l'observation temporelle suivie, continue et méticuleuse des transformations corporelles induites par l'expérience.

25 Pour une explicitation de ce point de vue constructiviste, cf. Daston 2000.

26 Barras 2000.

27 Loewy 1932. 
Le second exemple, graphiquement très proche du premier, a ceci de particulier qu'il consiste moins dans l'inscription d'une physiologie en soi que dans l'usage expérimental de tracés physiologiques pour donner une lisibilité graphique à une activité subjective spécifique. C'est le cas de l'usage de l'«hydrosphygmographe» par le psychiatre et criminologiste Cesare Lombroso. Cet instrument ne lui sert en effet pas, à proprement parler, à inscrire la pulsation cardiaque des «criminels» interrogés, mais à faire de celui-ci l'indicateur physiologique qui permet de donner une évidence visible à leur «subjectivité» par l'enregistrement des «modifications produites par les phénomènes purement psychiques: calculs, observations agréables ou pénibles, en entretenant le criminel d'évasion, de persécution, de jugement, etc.» ${ }^{28}$. Lombroso soumettra par exemple à ce test le jeune Prato Teonesto, «17 ans, fils d'alcooliques avec le type physionomique du criminel», dont les courbes sphygmographiques confirmeront l'anamnèse:

[II] commence à 5 ans à dérober du vin dans la cave de son père; il frappe plus tard par jalousie un de ses compagnons, abuse du vin et des femmes; la prison ne l'ennuie pas; il s'y trouve même très bien. ${ }^{29}$

Le sphygmographe révèle chez lui un «aplatissement du pouls» lorsqu'il sera confronté à la «photographie d'une femme nue» alors que «la vue du vin $\left(n^{\circ} 8\right)$, la vanité flattée $\left(n^{\circ} 9\right)$ élèvent puis abaissent la ligne du pouls». Grâce à l'hydrosphygmographe, les rythmes physiologiques se mettent ainsi à battre le tempo des désirs et des passions domestiqués dans l'espace de la mesure.

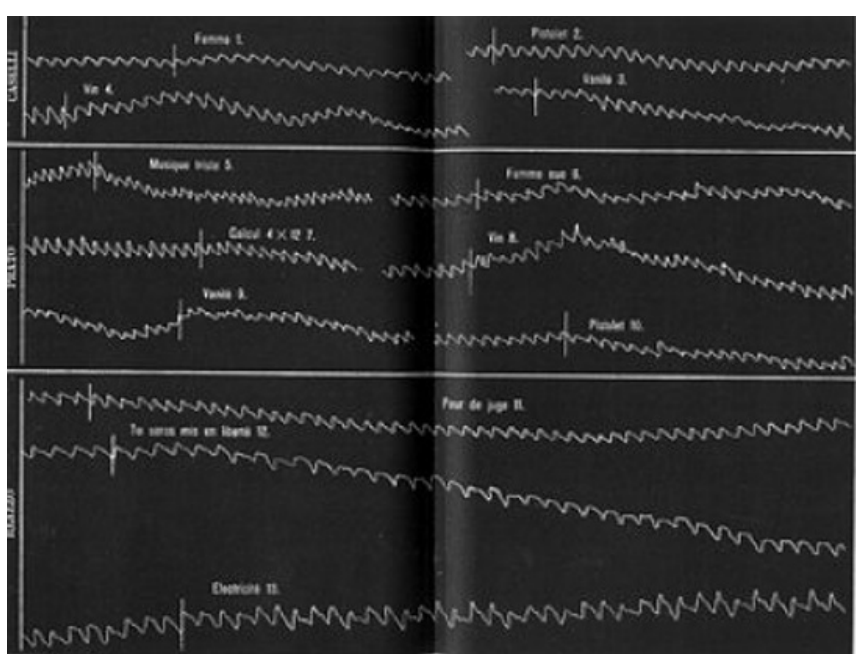

Fig. 5. «Résultat de quelques expériences avec l'hydrosphygmographe», dans: Cesare Lombroso, L'homme criminel (Paris 1895), 3 vol., Atlas, pl. XVII. Le cas de Prato se trouve au milieu.

28 Lombroso 1895 , vol. 1, 336.

29 Lombroso 1895, vol. 1, 339sq. Pour une analyse de ces «rhétoriques de la preuve» chez Lombroso, cf. Panese 2004. 


\section{Séquencer des transformations}

On trouve en médecine une autre forme de rhétorique visuelle des transformations des corps et des sujets. Elle consiste non plus à les inscrire dans un flux temporel continu, mais à fixer des séquences ou des phases de transformations temporellement organisées d'un même «objet» sur un même support. Généralement, il s'agit d'images animées qui permettent de rendre visibles - et plausibles - des transformations «naturelles» ou des transformations induites par l'intervention médicale ou expérimentale.

Une première catégorie peut être illustrée par des images récurrentes dans les traités d'obstétrique médicale, qui organisent la grossesse en phases significatives. Contrairement aux traités plus anciens qui privilégient avant tout, et selon une longue tradition, la représentation des positions fotales à la naissance, ici, l'objectivation par l'image de la transformation du corps féminin, de l'«état normal» au «9 $9^{\mathrm{e}}$ mois», témoigne de la «colonisation médicale» de l'ensemble de la grossesse à la fois comme objets de savoir et d'intervention sur un corps anonyme, sans visage, réduit au ventre et aux seins ${ }^{30}$.

Fig. 6. Jacques Pierre Maygrier, Nouvelles démonstrations d'accouchement (Paris 1822), pl. XXVI.

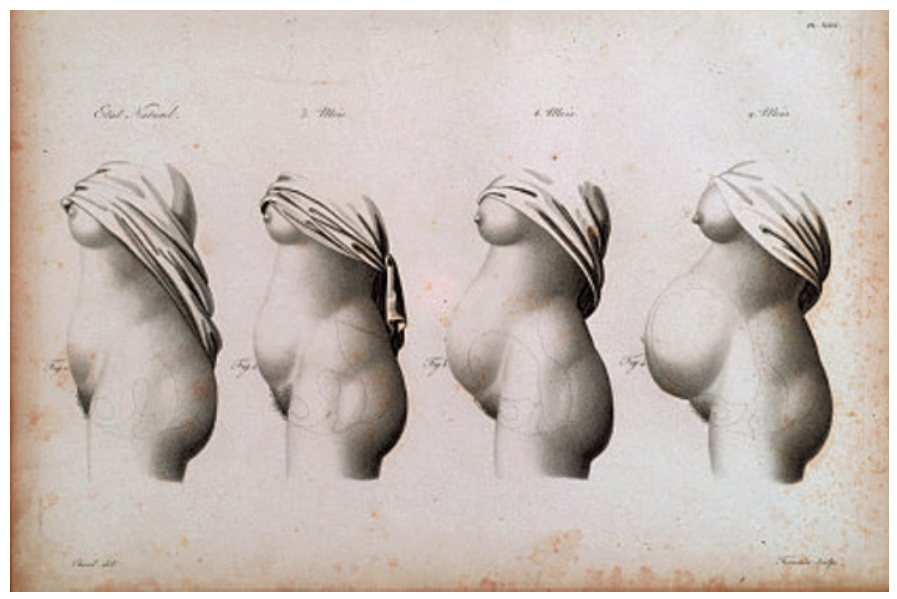

La même structure iconographique se retrouve dans des images qui saisissent des transformations induites expérimentalement. Un exemple canonique de cette catégorie est la célèbre gravure de William Harvey ${ }^{31}$. Simple en apparence, elle constitue un dispositif visuel qui permet de construire par l'image séquentielle l'évidence du flux unidirectionnel du sang comme indice d'une circulation sanguine, par la monstration de l'effet de l'action expérimentale élémentaire consistant à appuyer le doigt sur la veine d'un bras

30 Pour une lecture critique de cette «colonisation», cf. Duden 1991; 1996.

31 Harvey 1628. 
garrotté ${ }^{32}$. Comme dans le cas précédent, le réalisme de la description et la mise en scène du geste d'une main anonyme servent un effet de réel qui autorise tout spectateur à devenir l'expérimentateur virtuel de cette expérience.

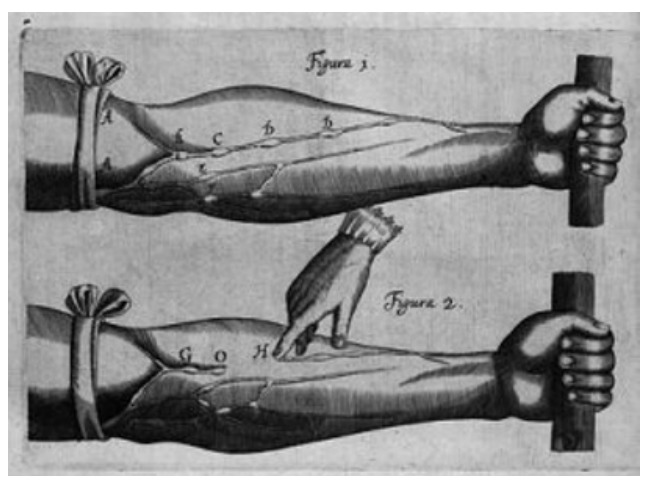

Fig 7. William Harvey, Exercitatio Anatomica de Motu Cordia et Sanguinis in Animali (Francfort 1628).

Ce genre de dispositifs visuels s'est largement banalisé en médecine car il permet, dans des domaines très divers, de mettre en évidence et de convaincre des résultats visibles d'interventions qui sont au cour de la pratique médicale. C'est le cas, de manière emblématique, en chirurgie reconstructive où le portrait séquencé s'est imposé à la fois comme style de représentation, comme outils d'analyse et comme moyen de persuasion.
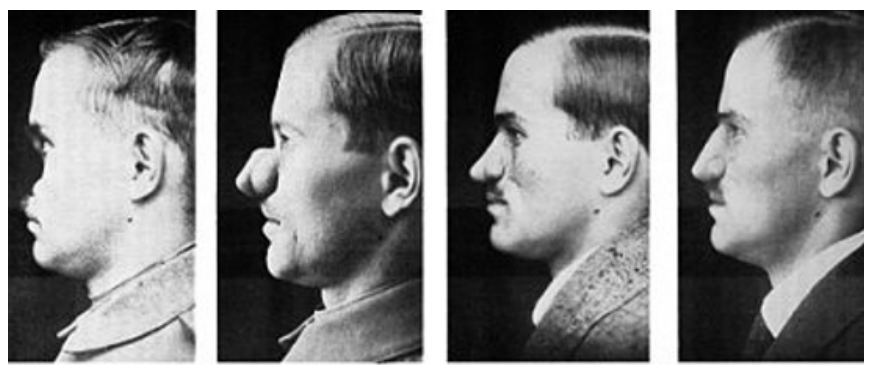

Fig. 8. Jacques Joseph, Nasenplastik und sonstige Gesichtsplastik, nebst einem Anhang über Mammaplastik (Leipzig 1931) ${ }^{1}$.

1 Pour une histoire de cette chirurgie spécifique, cf. Gilman 1999.

La construction par l'image de l'efficacité d'une action en termes d'avant et après a également recours au film, dans le cas de transformations difficiles à saisir par l'image fixe, tels les changements de comportements. Ceci peut être illustré par le documentaire étonnant que tournera John Huston en 1946 pour une commande de l'armée des Etats-Unis. On y découvre une psychiatrie militaire procédant à diverses «thérapies par la parole», dont l'hypnose, afin de lever les traumatismes de la seconde Guerre mondiale. On y découvre

32 Il convient de noter que cette image célèbre, empruntée par William Harvey à Girolamo Fabrizi d'Acquapendente, ne prouve pas à elle seule la circulation sanguine, mais constitue un des éléments qui lui permet de la postuler: le sens unidirectionnel du flux veineux est lié à la présence de valvules empêchant, dans les veines, le reflux du sang vers les extrémités. 

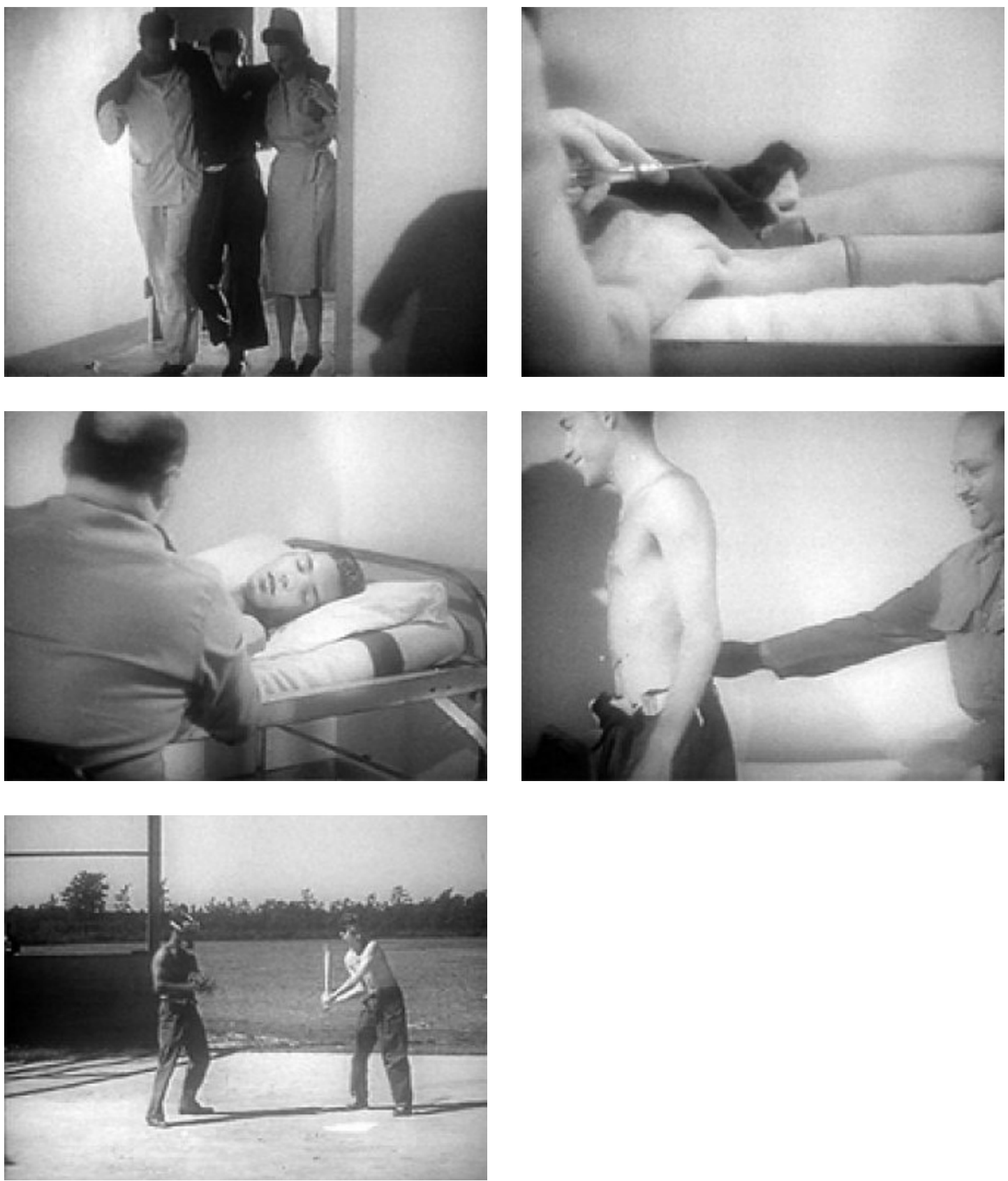

Fig. 9. Soldat souffrant d'une «paralysie hystérique», injection de Sodium Pentothal facilitant l'état d'hypnose, dialogue sous hypnose sur la raison du traumatisme, retour de la capacité de marcher sous hypnose, le soldat guéri à la fin du traitement. John Huston, Let There Be Light (USA 1946, 58 min.), U.S. Army Signal Corp Pictorial Service ${ }^{2}$.

2 Signalons un autre document intéressant sur le traitement par l'hypnose de traumatisme de guerre dont nous n'avons malheureusement pas identifié la source primaire, utilisé à titre illustratif dans le documentaire Sigmund Freud, l'invention de la psychanalyse, France 3 et La Sept-Arte, écrit par E. Roudinesco en collaboration avec Elisabeth Kapnist, réalisation Elisabeth Kapnist, (BFC production), 1997. Ces documents participent de l'histoire du «traumatisme» comme catégorie nosologique et socio-historique dans laquelle la psychiatrie militaire a joué un rôle important, très bien établie récemment par Fassin/Rechtman 2007. 
en particulier un soldat traumatisé, incapable de tenir son corps meurtri par des contractures, et bientôt remis sur pied par la suggestion hypnotique. Ce genre de films semble remplir une double fonction: donner une évidence visible à des effets thérapeutiques mais aussi exhiber une médecine capable de «miracles», en l'occurrence à la gloire de la Nation.

L'utilisation de l'image pour séquencer des transformations est, de manière évidente, d'autant plus récurrente que les domaines et les disciplines concernés intègrent ces transformations au cœur de leurs schèmes explicatifs. Ceci apparaît clairement dans ce que l'on pourrait appeler les «disciplines de la crise $»^{33}$ dont la psychiatrie constitue sans doute le modèle par excellence et ce, depuis sa constitution. Etienne Esquirol par exemple complète son traité «Des maladies mentales» par une série de figures mettent en scène l'inscription corporelle de la crise de «manie» et sa résolution par la «guérison».

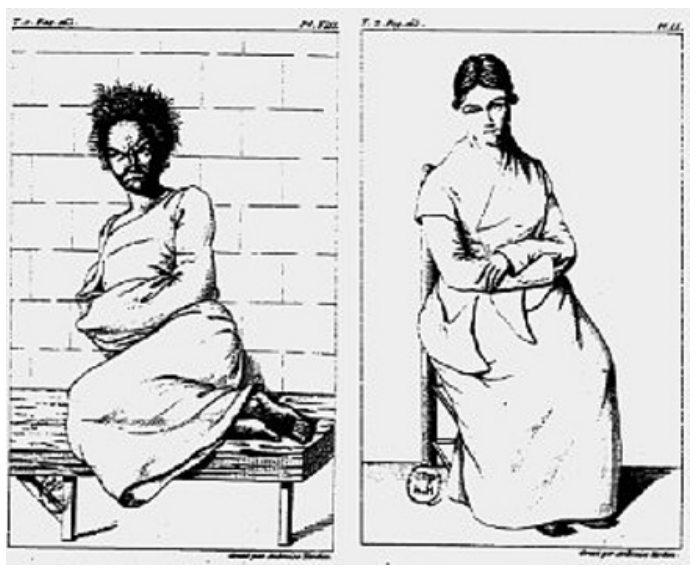

Fig. 10. «Maniaque, pendant l'accès et après la guérison». Esquirol, Etienne, Des maladies mentales considérées sous les rapports médical, hygiénique et médico-légal (Paris/ Londres/Lyon 1838).

Cette rhétorique visuelle permet une double opération. Elle institue tout d'abord par l'image la conception selon laquelle la «manie» est un état épisodique, contre l'idée d'une «folie complète»; corollairement, elle suggère qu'il est possible d'atteindre la raison du «maniaque» entre ces moments, afin d'opérer chez lui le retour stabilisé à la raison grâce au «traitement moral» ${ }^{34}$.

C'est dans cette tradition iconographique que s'inscrit également la mise en images de l'hystérie effectuée par le Service de photographie que JeanMartin Charcot instaura à la Salpêtrière ${ }^{35}$. Avant le film, la série photographique de la crise hystérique de la jeune patiente baptisée Augustine est transformée par l'image en une séquence régulière d'«attitudes» stéréoty-

33 Pigeaud 2006.

34 Swain 1997.

35 Cf. Bourneville/Régnard 1875-1878. 
pées qui, comme l'a bien montré Georges Didi-Huberman ${ }^{36}$, participe de «l'invention de l'hystérie» en en instituant le paradigme figuré. Ce dernier sera d'ailleurs formalisé par Paul Richer qui en inscrira les «périodes» sous la forme d'une syntaxe corporelle ${ }^{37}$. Comme dans les exemples précédents, mutatis mutandis, cette opération iconographique permet la «dépersonnalisation du sujet» par sa réduction à la lisibilité d'un corps générique.

Fig. 11. «Augustine», Désiré Magloire Bourneville et Paul Régnard, Iconographie photographique de la Salpêtrière (Paris 1875-1878).
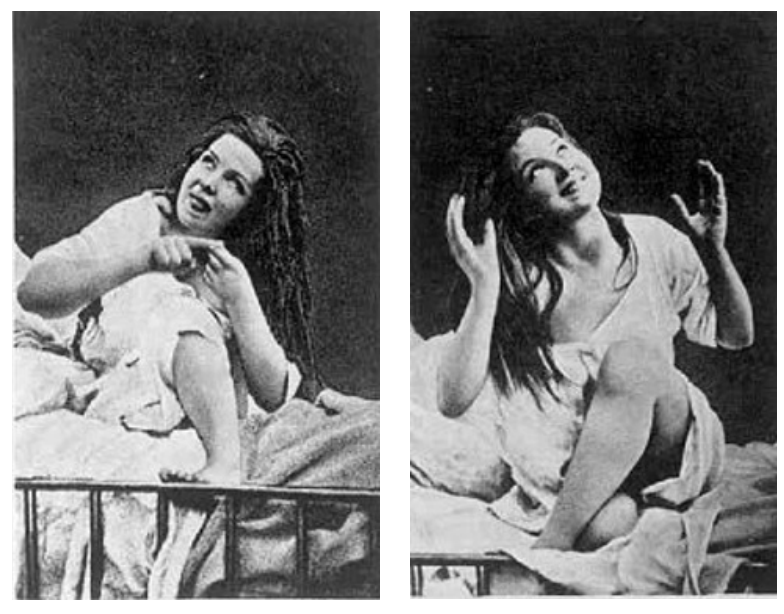

Fig. 12. Paul Richer, Etudes cliniques sur la grande hystérie ou hystéroépilepsie (Paris 1885).

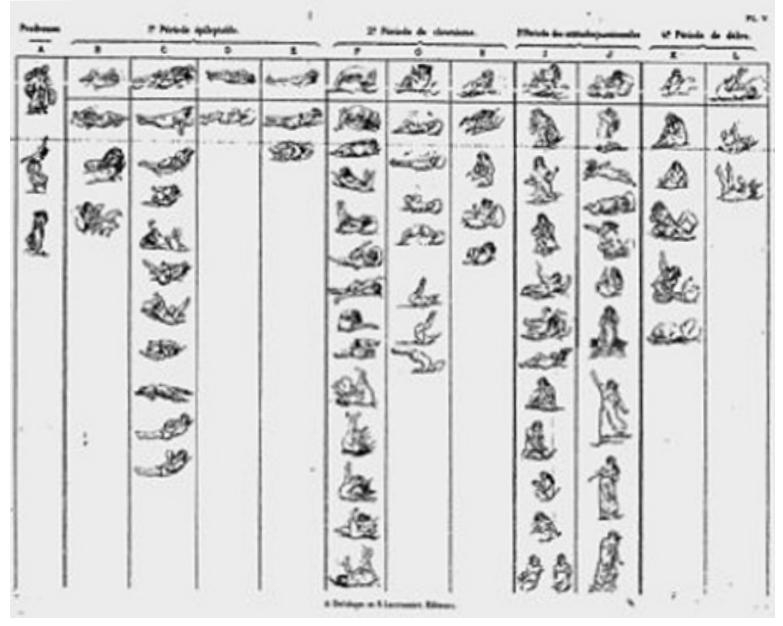

Une fois de plus, le film prendra, par d'autres moyens, le relais de cette même rhétorique de l'image. Dans La Nevropatologia (1908) ${ }^{38}$, Camillo Negro met en scène une patiente masquée à laquelle le neurologue semble suggérer la crise en l'interrogeant sur les symptômes qui généralement l'an-

36 Didi-Huberman 1982; Edelman 2003.

37 Richer 1885.

38 Publié dans le recueil de films Les incunables du cinéma scientifique, CNRS AV, ICS, 1984. Cf. Galloni 2002. On a pu voir dans ce film qui présente une dramatisation de la crise un exemple de l'origine scientifique des trames narratives du cinéma de fiction: Arnold 1994. 
noncent. La crise arrive aussitôt, la malade se débat sur un lit, contrainte par le neurologue et son assistant, suivie par un temps de léthargie puis par le «réveil» couronné par la pose des deux médecins aux allures fières entourant la malade.
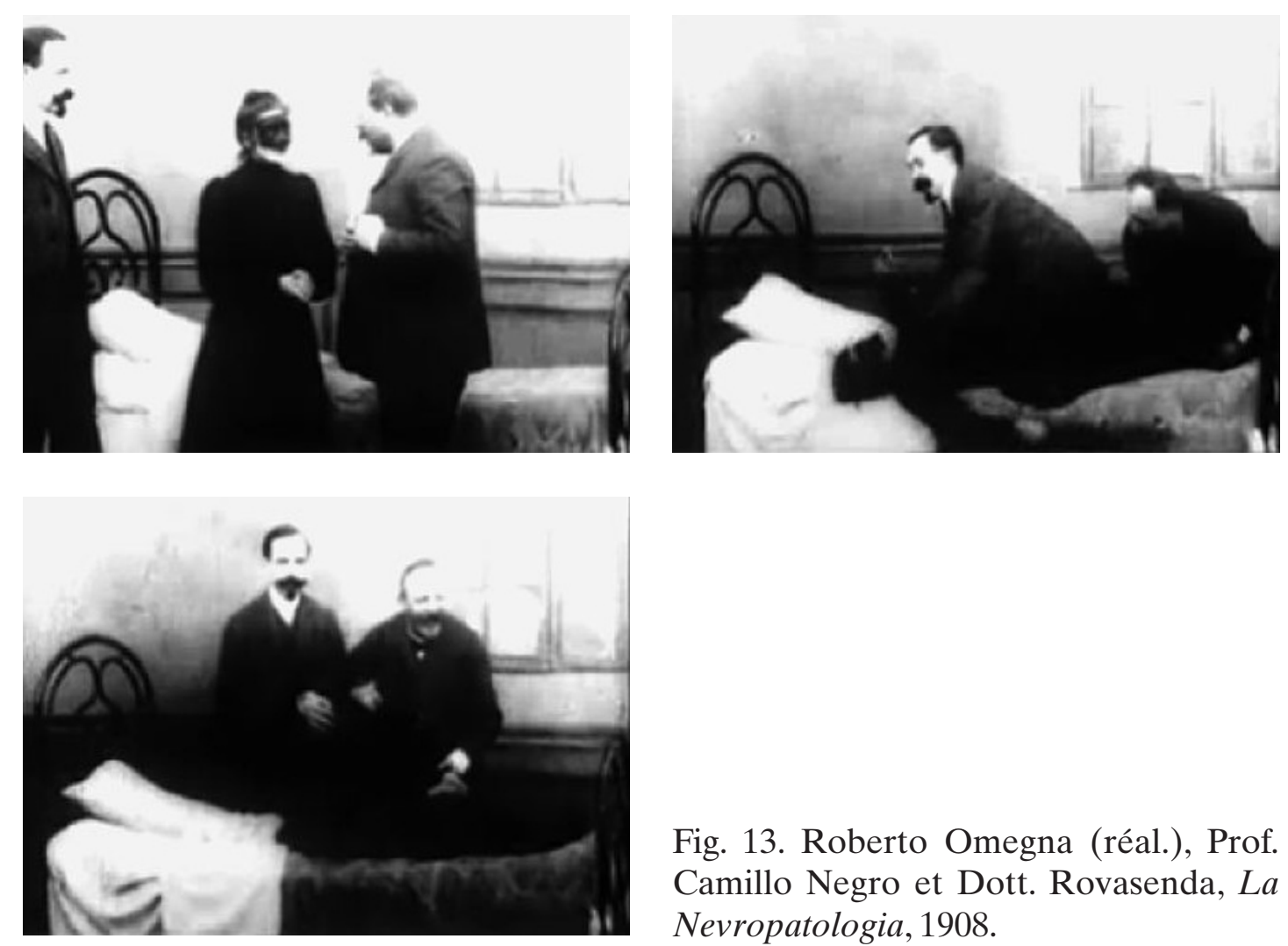

Fig. 13. Roberto Omegna (réal.), Prof. Camillo Negro et Dott. Rovasenda, La Nevropatologia, 1908.

Utilisés vraisemblablement comme outils pédagogique, comme le signale d'ailleurs Weiser, ces films - on l'imagine aisément - permettaient de «mobiliser la crise» au sein d'un auditoire, faisant ainsi l'économie d'une patiente réelle, pratique fort répandue depuis Charcot au moins. Mais ils permettaient aussi, comme dans le cas de la crise hystérique, de fixer un comportement dynamique stéréotypé. Cette rhétorique visuelle a ainsi contribué à documenter et, partant, à accréditer une «mise en corps» de la «névropathologie» comme déliée de la subjectivité de celle qui l'incarne - nous ne savons rien d'elle -, une conception qui sera ébranlée par la psychanalyse: «Les recherches psychanalytiques de Freud», se souvient Sandor Ferenczi, «nous ont appris à considérer les symptômes de la conversion hystérique comme des représentations, par le corps, de fantasmes inconscients.» ${ }^{39}$ Sans supposer ici la supériorité épistémologique de l'une ou de l'autre de ces versions de la

39 Ferenczi 1919. 
«crise», signalons simplement que la visualité qui est cœur de ces dispositifs peine à rendre intelligibles les contenus subjectifs des symptômes corporels manifestes de ces femmes en souffrance, contenus écartés au profit de la construction par l'image de la crise comme processus «naturel» aux formes stables et récurrentes.

Notons sur ce point que l'arrivée du film sonorisé permettra d'étendre son usage à bien d'autres domaines relevant de l'observation des comportements. Mentionnons à titre d'exemple, et toujours dans le domaine «psy», les films réalisés par Jean Piaget et Bärbel Inhelder, à l'occasion de leurs travaux sur la «logique de l'enfant $»^{40}$. Ces expériences filmées sont sans doute apparues aux deux psychologues comme un moyen idéal pour fixer par l'image les dispositifs expérimentaux (des tests logiques d'aptitude), le dialogue entre l'expérimentateur et son sujet (la communication des consignes) et surtout les performances de ce dernier, matérialisées et visualisées par leurs manipulations, et dont la version filmée permettait la comparaison, en fonction de l'âge du sujet. Dans ce cas, la rhétorique visuelle permet de construire dans
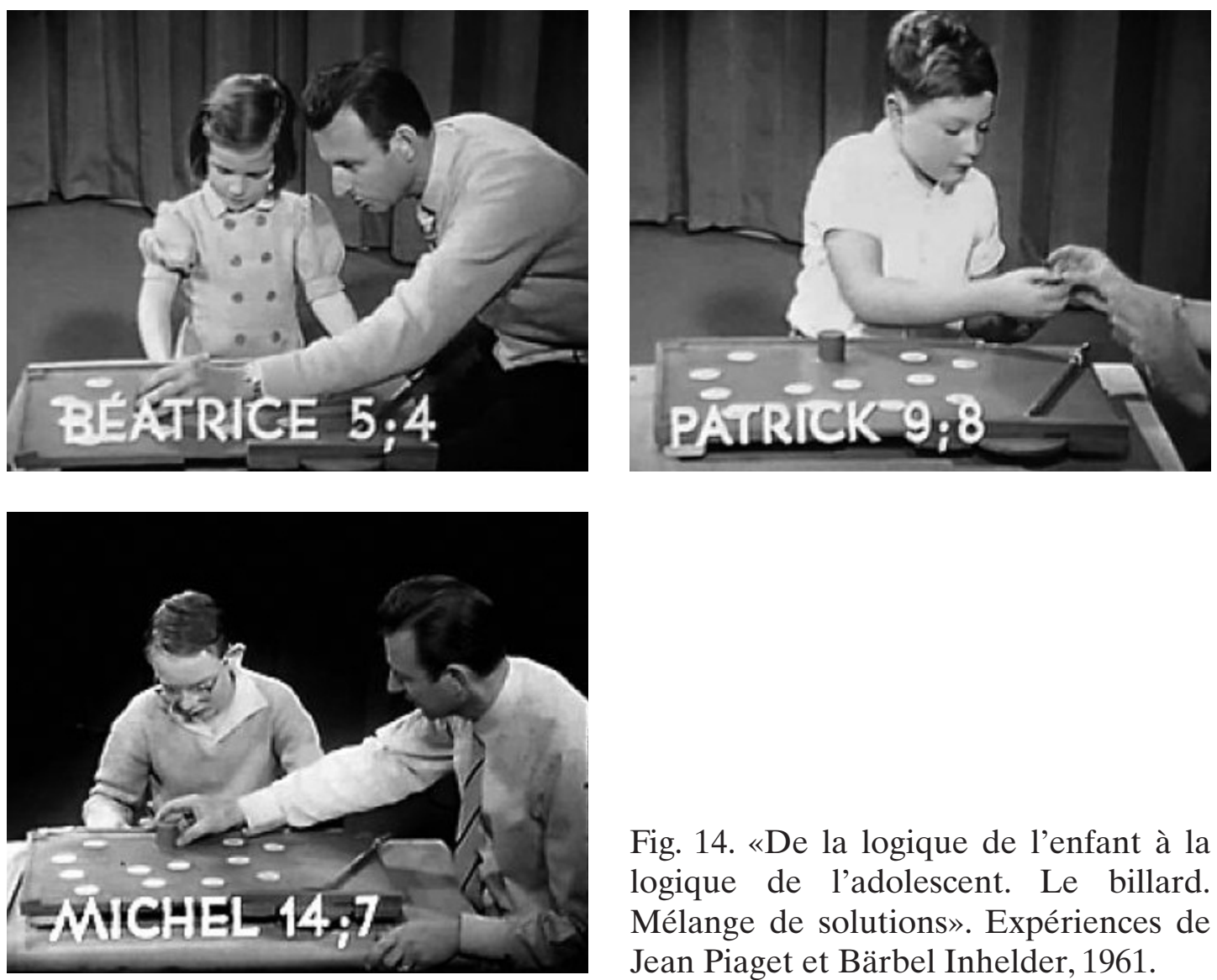

Fig. 14. «De la logique de l'enfant à la logique de l'adolescent. Le billard. Mélange de solutions». Expériences de Jean Piaget et Bärbel Inhelder, 1961.

40 Cf. le chapitre «L'égalité des angles d'incidence et de réflexion et les opérations d'implication réciproque» dans Inhelder/Piaget 1955, précisément basé sur ces expériences filmées. 
un même mouvement une archive de dispositifs expérimentaux, des relevés de comportements cognitifs, un outil de démonstration scientifique et pédagogique et, de manière plus générale, de fonder de manière «vériste» et «expérimentale» la célèbre hypothèse piagétienne des stades du développement de la pensée chez l'enfant et l'adolescent.

\section{Explorer l'intérieur des corps}

Les images animées que nous avons parcourues jusqu'ici sont le fruit d'une opération épistémique qui consiste à capturer un phénomène de transformation par son inscription technique dans un espace figuratif structuré temporellement, qu'il s'agisse du mouvement des corps, de la réaction de sujets à des stimulations, de rythmes physiologiques, de transformations corporelles, du résultat d'actes expérimentaux ou thérapeutiques, de mutations comportementales ou encore du déroulement d'expériences. Si vaste que soit le corpus documentaire couvert par cette acception de l'image animée, force est de constater qu'il exclut encore celles dont la pertinence médicale découle moins de la trame temporelle à laquelle leur référent est soumis que simplement de la possibilité technique de donner à voir l'invisible et en direct l'intérieur des corps vivants.

Rappelons, à l'instar de Weiser, que le premier spectacle animé de l'intérieur du corps a été rendu possible par la médiation technique de la radiologie. En effet, si elle consiste généralement aujourd'hui à fixer l'image produite

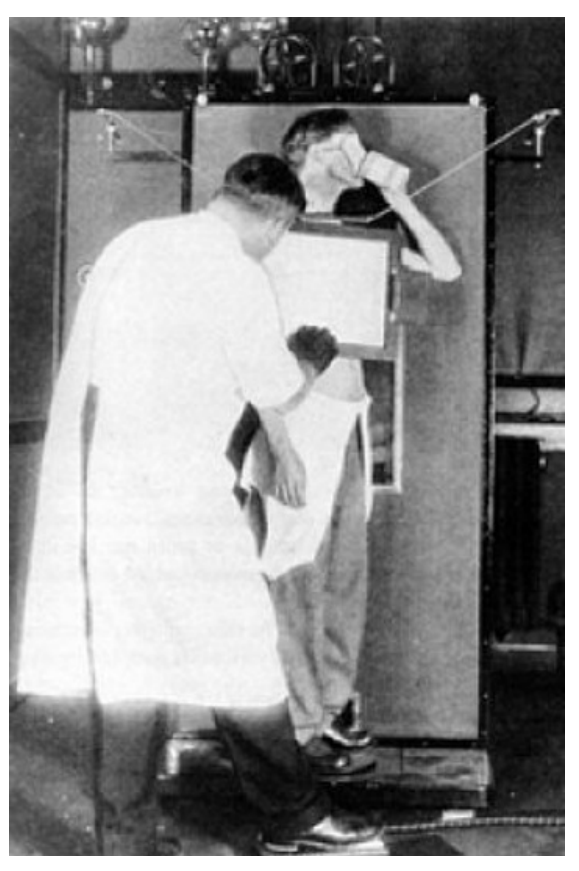

Fig. 15. Russel, D., «Carman au fluoroscope examinant la trajectoire d'un liquide opaque dans l'œsophage d'un patient», dans: R. D. Carman/ A. Miller, The Roentgen Diagnosis of Disease of the Alimentary Canal (Philadelphia 1917). 
par les Rayons X sur un support, les premiers dispositifs permettaient littéralement d'ouvrir une fenêtre vers l'intérieur du corps, l'une et l'autre pouvant se mouvoir, afin de présenter à l'œil du médecin ou du radiologue telle ou telle de ses parties ${ }^{41}$. Du point de vue du spectateur, tout se passait alors comme s'il était face au film «en direct» de l'exploration de corps transparents.

C'est d'ailleurs ce spectacle que des radiologues comme Robert Janker, professeur de radiologie à Bonn, commencent à filmer dès le début des années 1930, initiant le genre de la «Röntgenkinematographie» ${ }^{42}$, à des fins documentaires et de démonstrations.
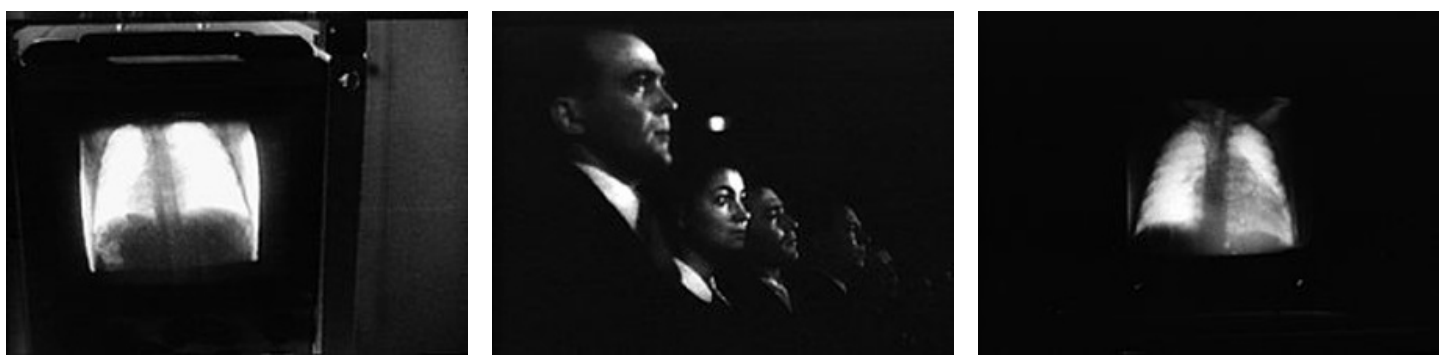

Fig. 16. Patient sous le fluoroscope vu par l'objectif de la camera et projection publique du film. Robert Janker, Beispiele aus Röntgen-Kinofilmen beim Menschen (s.d., années $1930)^{3}$.

3 Ces documents (film 16 mm transférés sur vidéo) proviennent du Deutsches Röntgen Museum de Remscheid qui a eu la générosité de les mettre à notre disposition pour l'exposition $X$ Regard vers l'intérieur, Fondation Claude Verdan, Lausanne, 2002.

Dans ces documents surprenants, la pertinence médicale réside dans la possibilité d'une anatomie dynamique des corps vivants. C'est d'ailleurs l'une des raisons qui expliquent la très rapide hybridation de cette innovation avec la chirurgie, autre discipline médicale de l'accès à l'intérieur des corps. Dès la première Guerre mondiale, marquée par les premiers usages systématiques de l'artillerie et des obus à fragmentation, les blessés sont même «opérés, raconte Marie Curie, sous le contrôle des rayons» ${ }^{43}$. Cette pratique, née à un moment où l'on sous-évalue la toxicité des rayons, marque le début d'un développement qui prendra son essor bien plus tard avec d'autres moyens. De la laparoscopie à ses nombreux succédanés, les interventions chirurgicales et de très nombreux diagnostics se font désormais par la médiation de l'image animée, rendue possible en particulier par le développement parallèle de la vidéo, de la miniaturisation des optiques et de la conduction de la lumière.

41 Pasveer 1993.

42 Janker 1939.

43 Curie 1921, citée dans Bordry/Fayard 1992. 
Relativement simple dans sa version classique - les premiers développements de l'endoscopie datent du début du XIX ${ }^{\mathrm{e}}$ siècle -, le geste médical assisté est aujourd'hui en passe d'instaurer la médiation par l'image animée comme point de passage obligé de l'intervention sur les corps. Une des caractéristiques générales de ses récents développements est la délocalisation. Délocalisation du geste tout d'abord, dont l'action ne se porte plus directement sur le corps, mais sur une interface qui le traduit, l'assure, et le transmet à des instruments robotiques qui effectuent la tâche. Délocalisation respective du patient et du médecin ensuite, leur co-présence étant médiatisée par l'image interactive, que ce soit à quelques mètres de distance ou, comme dans l'opération de télé-chirurgie «Lindbergh» ${ }^{44}$, des deux côtés de l'Atlantique.
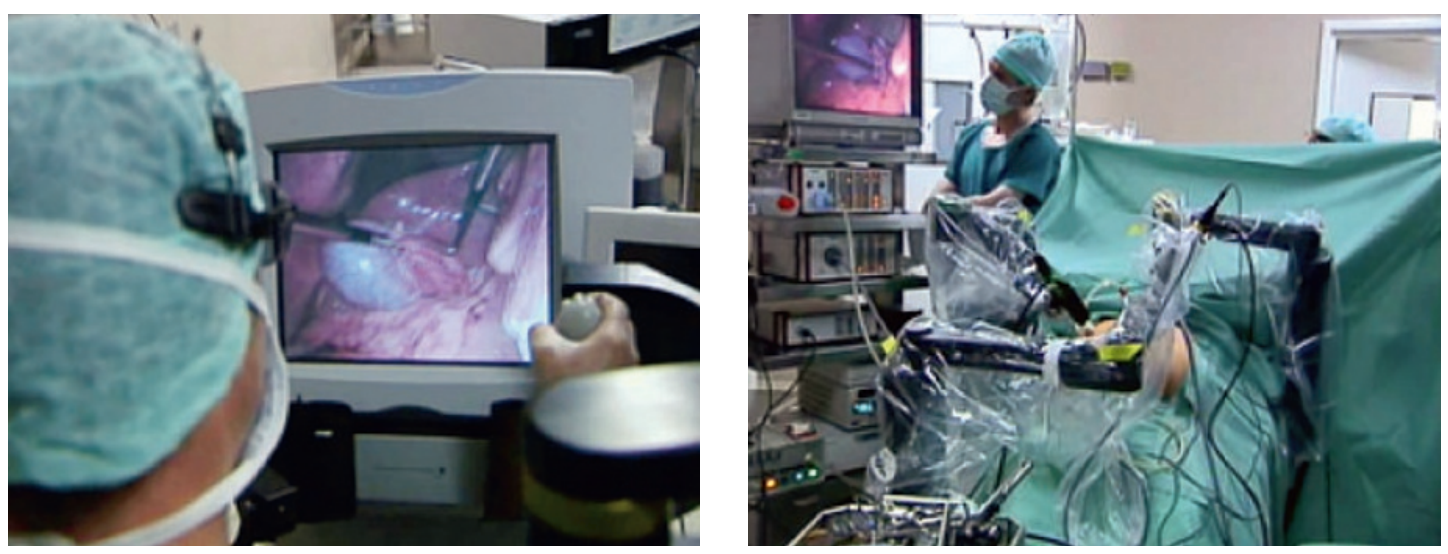

Fig. 17. Chirurgien au télémanipulateur et surveillance de l'opération laparoscopique par écran. Robotisation du geste chirurgical, H. Vuilleumier, N. Halkic, Lausanne, CEMCAV-CHUV, 2003.

Aujourd'hui, sur les établis des ingénieurs, se prépare une nouvelle intégration entre techniques d'intervention et imagerie médicale ${ }^{45}$. Son principe consiste à superposer l'image réelle et actuelle du patient à sa représentation virtuelle et différée, afin de guider le geste de l'opérateur et lui permettre d'éviter, par le phénomène dit de «retour de force» contrôlé par le robot, qu'il ne s'aventure dans des zones dangereuses. Sans entrer dans le détail

44 L'«Opération Lindbergh», dont le nom renvoie bien sûr au pionnier américain de l'aviation qui a traversé l'Atlantique pour la première fois en 1927, a été réalisée par Jacques Marescaux et son équipe en 2001 entre New York, où se trouvait le chirurgien, et Strasbourg, où se trouvait la patiente.

45 Cette ligne de recherche est de plus en plus développée, au point qu'un journal qui lui est entièrement dédié a vu le jour en 2006 chez l'éditeur allemand Springer: International Journal of Computer-Assisted Radiology and Surgery. 
technique de ces développements, retenons ici qu'ils marquent moins l'achèvement d'une supposée «virtualisation» du corps, que l'inauguration d'un espace visuel dynamique d'un genre nouveau, alliant le réel et le virtuel, la matérialité du corps et ses images, la représentation et l'intervention, le voir et le faire.

\section{Orienter des comportements}

Il est parfois difficile de connaître précisément les publics auxquels s'adressaient les documents que nous avons commentés. On peut toutefois avancer sans trop risquer de se méprendre que la plupart d'entre eux relèvent de l'ésotérisme médical, par opposition à l'exotérisme qui caractérise les images et les films qui ont été délibérément réalisés pour un public de non-spécialistes. C'est par définition à cette seconde catégorie qu'appartiennent les documents de la tradition du cinéma sanitaire ${ }^{46}$. Le film y est un instrument qui vise à transformer les comportements relatifs à la santé. Sur le plan du genre, il s'agit très souvent de mises en scène véristes et dramatiques de comportements néfastes à la santé qui produisent des effets de vérité, de réalité et de conviction qui participent à l'intériorisation de codes, de normes et de représentations soit, d'une manière générale, à la construction du «soi objectif $»^{47}$ des spectateurs.

Comme l'indiquait Weiser, les films sanitaires doivent être considérés comme médicaux à part entière et ce, non seulement parce qu'ils traitent de thématiques médicales, mais surtout parce qu'ils participent d'une rhétorique médicale, même si elle prend souvent le chemin de la narration fictionnelle. Ceci peut être illustré par le film Misères de femmes - Joies de femmes de 1929 supervisé par le célèbre Sergei Mikhailovich Eisenstein ${ }^{48}$.

46 Sur le cinéma sanitaire, cf. Lefebvre 1993; 2002a.

47 L'expression est empruntée à Dumit 1998, 84: «Objective self-fashioning is thus an acknowledgment of local mutations in categories of persons highlighting the active and continual process of self-definition and self-participation in that process. Objective self-fashioning is how we take facts about ourselves - about our bodies, our minds, our capacities, traits, states, limitations, propensities, etc. - that we have read, heard or otherwise encountered in the world, and incorporate them into our lives.»

48 Le titre original allemand est Frauennot - Frauenglück, par Grigori Aleksandrov, Edouard Tissé, Sergei Mikhailovich Eisenstein, Production Praesens-Film AG, Zurich, 1929, 55 min. Pour une analyse de l'histoire mouvementée de la production de ce film expliquant notamment les différents montages dont il a fait l'objet et les messages contrastés qu'ils ont servis, nous renvoyons à la reconstitution précise de Albera 2002. 
Il importe de livrer quelques éléments pour comprendre l'histoire racontée. Il s'agit de portraits de femmes qui se succèdent, sauf une, au portillon du malheur. Une mère de famille pauvre est enceinte et l'on nous fait comprendre qu'une bouche de plus à nourrir serait une bouche de trop. Elle s'en va chez une faiseuse d'anges dont elle sortira presque morte, exsangue pour cause d'hémorragie. L'art médical de l'hôpital dont on exhibe la propreté et la rigueur sauvera cette victime de l'avortement clandestin. Une autre femme est heureuse d'annoncer sa grossesse à son compagnon grutier, lequel tombe de la grue et meurt sous ses yeux, la voix hors-champ annonçant que, comme des milliers de femmes, la voici devenue une nouvelle candidate à l'avortement clandestin car elle ne saurait garder seule cet enfant. Dans un cadre plus bourgeois cette fois, un aventurier charmeur et volage contraint sa victime d'amours sans lendemains à se rendre chez le médecin en quête d'un avortement qui lui sera refusé car «interdit». Une autre femme qui lui succède au cabinet médical se fait prescrire une césarienne. La dernière partie du film est consacrée aux destins de ces deux femmes. La première trouvera la mort chez la faiseuse d'anges à la suite des «pratiques brutales» de cette dernière, la seconde deviendra mère à l'hôpital. Cette longue séquence à la tonalité dramatique fait alterner l'art médical, avec son calme, sa clarté, son outillage perfectionné, son personnel formé, son hygiène irréprochable et sa performance thérapeutique, et l'avorteuse pratiquant derrière des persiennes closes, avec une aiguille à tricoter, épongeant le sang avec un vulgaire torchon et envoyant la jeune femme à la mort. Le film se
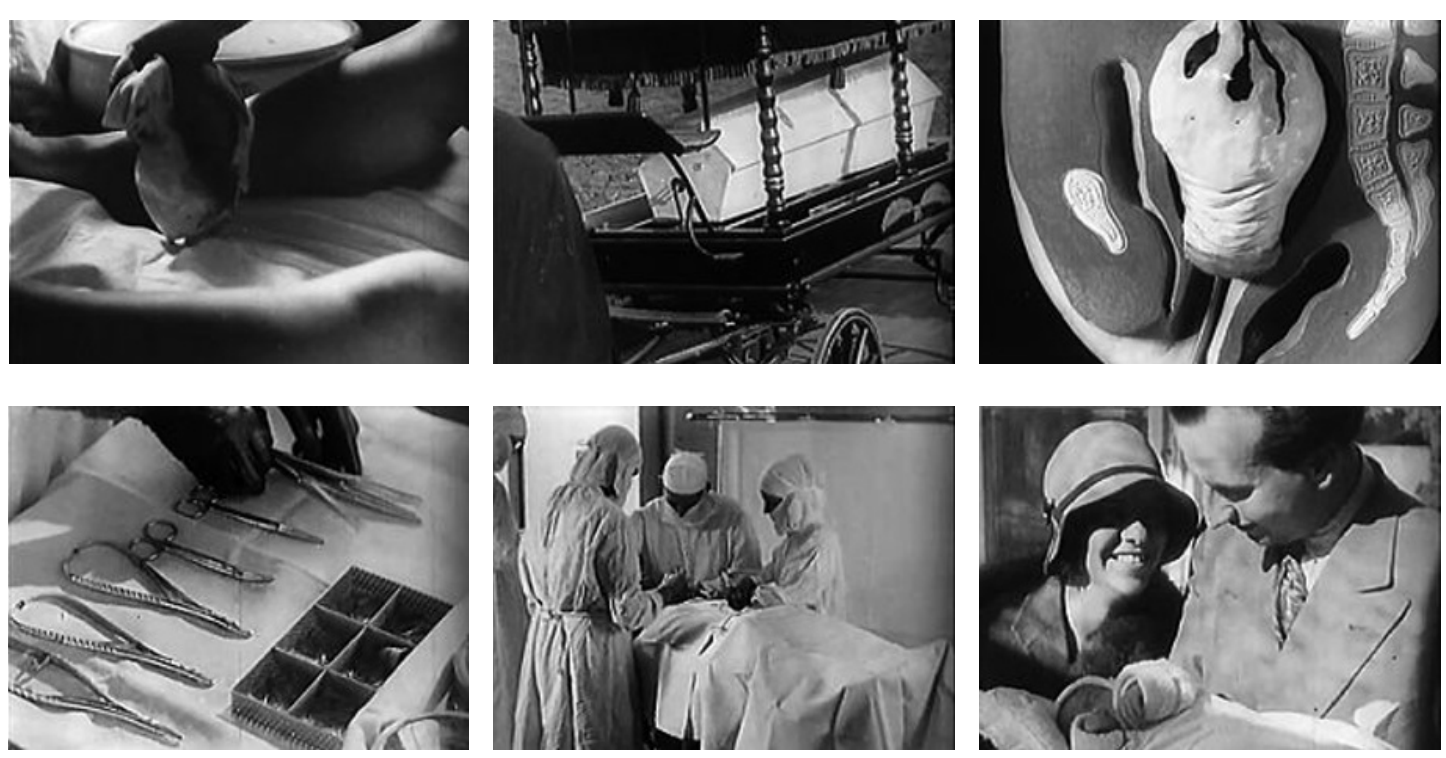

Fig. 18. Chez la faiseuse d'anges et à l'hôpital, Frauennot - Frauenglück, par Grigori Aleksandrov, Edouard Tissé, Sergei Mikhailovich Eisenstein, Production PraesensFilm AG, Zurich 1929. 
termine par le convoi funèbre de la première et l'heureuse sortie de l'hôpital de la seconde, devenue mère, avec son mari et son enfant ${ }^{49}$.

L'efficacité rhétorique de ce genre de film semble proportionnelle à la simplicité de son scénario. Celui-ci reproduit, en en soulignant les passages, les trois moments de la structure dramatique classique: la situation initiale et l'incident déclencheur, le développement du nœud dramatique et le dénouement, selon une opposition entre le bien et le mal. La simplicité de cette trame traduit pourtant mal la complexité de ce film. A y regarder de près, on s'aperçoit que son efficacité est aussi intimement liée à une rhétorique visuelle de la démonstration inspirée par la médecine. Celle-ci est lisible d'emblée dans sa structure: comme dans une série de leçons cliniques, le film procède explicitement par la présentation de «cas» qui sont construits par l'image et par la voix hors-champ du discours autorisé de la médecine comme emblématiques de destins «typiques» de femmes conduites vers l'avortement par les drames de l'existence. A cet inventaire illustré succède une narration comparative qui, comme dans une discussion technique, vise la démonstration en comparant point par point et de manière tranchée les pratiques légitimes et illégitimes, l'art de la médecine qui soigne et qui guérit et les pratiques dangereuses des avorteuses, en glissant entre les interstices des drames qui se nouent l'explication sur des modèles anatomiques des causes des hémorragies entraînant le décès des malheureuses victimes. En d'autres termes, si dans ces films le drame est bien présent, fictionnalisé par le jeu des comédiennes, il sert avant tout une démonstration reposant sur une logique empirique implacable servie par le réalisme des scènes et les nombreuses occasions de s'identifier avec elles. Il est enfin surprenant de relever que, malgré le thème traité, la «morale» en matière d'avortement est comme suspendue et le destin dramatique de ces femmes exposé avec bienveillance. Il nous semble pouvoir déceler en cela l'affirmation de la modernité d'une médecine qui suspend ses jugements moraux, ou plutôt qui leur substitue la promotion de l'acte technique comme solution «démocratique» à cette catégorie du malheur des femmes.

49 Notons que cette trame narrative semble avoir été à l'origine même du projet. «A en croire Lazare Wechsler lui-même [producteur qui lança le projet], dans ses mémoires écrits dans les années 60 et selon son témoignage oral par la suite, il eut l'idée de ce film en confrontant la nouvelle, souvent lue dans les journaux à la rubrique des faits divers, de la mort d'une femme des suites d'un avortement clandestin, à l'ouverture d'une nouvelle clinique gynécologique ultra-moderne à Zürich, sous la direction du Dr Walthar.» (Albera 2002, 74) 


\section{Ouverture}

La rhétorique visuelle des images médicales est inséparable d'une praxis des corps. A chaque étape de ce parcours, nous avons en effet tenté de montrer qu'elle ne se limite pas à les «représenter», mais qu'elle en façonne littéralement des versions spécifiques, même lorsqu'ils semblent s'offrir «immédiatement» au regard: le corps en mouvement, le corps physiologique, le corps réparé, le corps expérimental, le corps en santé et, plus généralement, le corps et le sujet en action.

Ce façonnage visuel des corps s'inscrit sans doute dans le fil d'une histoire technicienne de l'ocularocentrisme ${ }^{50}$ médical, marquée par des technologies visuelles qui coexistent souvent et se mêlent. $\mathrm{Si}$, en médecine, les corps naissent souvent «par les images», nous avons vu que leur animation nécessite des ruses techniciennes, figuratives, rhétoriques et narratives qui permettent d'inscrire leurs transformations sur la rétine du médecin ou du chercheur ${ }^{51}$. La variété de ces ruses mène au constat que le «film», stricto sensu, n'est qu'un moyen parmi d'autres pour parvenir à rendre compte de la dimension dynamique et temporelle des phénomènes corporels. Elle montre aussi que les aspects techniques servent des opérations épistémiques et que les technologies visuelles d'une discipline témoignent toujours de ses schèmes explicatifs. En médecine, l'image animée découle de l'affirmation de la temporalité comme clé de lecture des corps et en même temps la rend possible. Derrière cette apparente évidence se cache l'historicité même de l'épistémologie de la médecine: si l'on admet que le temps est une variable cardinale de l'expérimentation, le développement de l'image animée en médecine marquerait ainsi un moment décisif de la modernité médicale. D'une manière plus générale, cela implique qu'une façon de faire l'histoire de la médecine consiste dans l'analyse de ses cultures visuelles, historiquement de plus en plus marquées par la pluralité et la diversité des médiations scopiques qui lui permettent de construire la visualité, la pertinence et l'intelligibilité de ses objets.

50 Le terme est de Martin Jay. Cf. Jay 1993a; 1993b.

51 Nous reprenons ici la célèbre formule «rétine du savant»par laquelle l'astronome Jules Janssen caractérisait la photographie, formule reprise par Lynch 1985. 


\section{Bibliographie}

Albera, François, «Un destin mouvementé: Frauennot-Frauenglück», dans: Rémy Pithon (dir.), Cinéma suisse muet: lumières et ombres (Lausanne 2002) 73-84

Arnold, Jean-Michel, «La grammaire cinématographique: une invention des scientifiques», dans: Alexis Martinet (coord.), Le cinéma et la science (Paris 1994) 210-217

Barras, Vincent, «Physiologie et thérapeutique alpines au tournant du XX $\mathrm{X}^{\mathrm{e}}$ siècle», dans: JeanClaude Pont/Jean Lacki (éds), Une cordée originale (Genève 2000) 219-233

Bordry, Monique/Françoise Fayard, «Les rayons X sortent de l'anonymat», Journal de l'Institut Curie 21 (1992) [reproduit dans: http://www.curie.u-psud.fr]

Bourneville, Désiré Magloire/Paul Régnard, Iconographie photographique de la Salpêtrière (Paris 1875-1878)

Braun, Marta, Picturing Time: The Work of Etienne-Jules Marey (1830-1904) (Chicago 1994)

Chalvon-Demersay, Sabine, «La confusion des conditions. Une enquête sur la série télévisée Urgences», Réseaux 95 (1999) 236-283

Curie, Marie, La Radiologie et la Guerre (Paris 1921)

Dagognet, François, Etienne-Jules Marey: la passion de la trace (Paris 1987)

Daston, Lorraine, «The Coming into Being of Scientific Objects», dans: Lorraine Daston (ed.), Biographies of Scientific Objects (Chicago/London 2000)

- /Peter Galison, Objectivity (New York/Cambridge, Mass. 2007)

- /Peter Galison, "Image of Objectivity", Representations 40 (1992) 81-128

Didi-Huberman, Georges, Invention de l'hystérie: Charcot et l'iconographie photographique de la Salpêtrière (Paris 1982)

Duden, Barbara, L'invention du foetus (Paris 1996)

- The Woman Beneath the Skin: A Doctor's Patients in Eighteenth-Century Germany (Cambridge, Mass. 1991)

Dumit, Joseph, "A Digital Image of the Category of the Person: PET Scanning and Objective Self-Fashioning", dans: Gary Lee Downey/Joseph Dumit (eds), Cyborgs \& Citadels: Anthropological Interventions in Emerging Sciences, Technologies and Medicines (Santa Fe 1998) 83-102

Edelman, Nicole, Les métamorphoses de l'hystérique: du début du XIX siècle à la Grande Guerre (Paris 2003)

Fassin, Didier/Richard Rechtman, L'Empire du traumatisme. Enquête sur la condition de victime (Paris 2007)

Ferenczi, Sandor, «Phénomènes de matérialisation hystérique» (1919), dans: Psychanalyse 3, Euvres complètes (Paris 1974), tome III, 1919-1926, 53-65

Frayling, Christopher, Mad, Bad and Dangerous? The Scientist and the Cinema (London 2005)

Galilei, Galileo, Istoria e dimostrazioni intorno alle macchie solari e loro accidenti comprese in tre lettere scritte all'illustrissimo Signor Marco Velseri Linceo, Duumviro d'Augusta Consigliero di Sua Maesta Ceserea dal Signor Galileo Galilei Linceo, Nobil Fiorentino, Filosofo, Matematico Primario del Sereniss. D. Cosimo II. Gran Duca di Toscana (Roma 1613)

Galloni, Marco, «L'histoire du cinéma scientifique à l'université de Turin», Bulletin de la Sémia 2 (2002) 5-6

Gilman, Sander L., Making the Body Beautiful (Princeton 1999)

Harper, Graeme/Andrew Moor (eds), Signs of Life: Cinema and Medicine (London 2005)

Harvey, William, Exercitatio Anatomica de Motu Cordia et Sanguinis in Animali (Francofurti 1628)

Inhelder, Bärbel/Jean Piaget, De la logique de l'enfant à la logique de l'adolescent (Paris 1955)

Janker, Robert, Die Röntgenkinematographie (Stuttgart/Berlin 1939)

Jay, Martin, «Les régimes scopiques de la modernité», Réseaux 61 (1993a) 101-112

- Downcast Eyes: The Denigration of Vision in Twentieth-Century French Thought (Berkeley 1993b)

Latour, Bruno, «Les 〈vues〉 de l'esprit. Une introduction à l'anthropologie des sciences et des techniques», Culture Technique 14 (1985) 5-29

Lefebvre, Thierry, «Cinéma et hygiène. Les débuts d'une fructueuse collaboration» dans: Didier Nourrisson (dir.), Education à la santé. XIX $X^{e}-X X^{e}$ siècle (Rennes 2002a) 71-81

- «Eugène-Louis Doyen (1859-1916)», Bulletin de la Sémia 2 (2002b) 1-3 
- «Le docteur Doyen, un précurseur», dans: Alexis Martinet (coord.), Le cinéma et la science (Paris 1994)

- «Le cinéma contre la syphilis. Pré-enquête sur une lignée cinématographique» dans: Emmanuelle Toulet/Christian Belaygue (dir.), Catalogue du $3^{e}$ Festival CinéMémoire (Paris 1993) 160-174

- La chair et le cellulö̈d: le cinéma chirurgical du docteur Doyen (Brionne 2004)

Loewy, Adolf, Physiologie des Höhenklimas (Berlin 1932)

Lombroso, Cesare, L'homme criminel: criminel-né, fou moral, épileptique, criminel fou, criminel d'occasion, criminel par passion: étude anthropologique et psychiatrique (Paris 1895)

Lynch, Michael, «La rétine extériorisée: sélection et mathématisation des documents visuels», Culture technique 14 (1985) 108-123

Marey, Etienne-Jules, La méthode graphique dans les sciences expérimentales et particulièrement en physiologie et en médecine (Paris 1878)

O'Connor, Michael, «The Role of the Television Drama ER in Medical Student Life: Entertainment or Socialization?», JAMA 280 (1998) 854-855

Panese, Francesco, «Sur les traces des criminels. L'institution «scientifique〉 de la différence chez Cesare Lombroso», dans: Véronique Mauron/Claire de Ribaupierre (éds), Les figures de l'idiot (Paris 2004) 107-116

- «Disciplines scientifiques et disciplines du regard au XVII ${ }^{\mathrm{e}}$ siècle», Réseaux 94 (1999) $149-171$

Pasveer, Bernike, «Images et objets: la tuberculose et les rayons X», Techniques et Culture 25-26 (1995) 1-18

- «Fabriquer le corps. Image radiologique et médecine», Traverses 8 (1993) 82-90

Peneff, Jean, «La face cachée d'Urgences, le feuilleton de la télévision», Genèse 29 (1998) 122-145

Pierantoni, Ruggero, Forma fluens: il movimento e la sua rappresentazione nella scienza, nell'arte e nella tecnica (Torino 1986)

Pigeaud, Jackie, La crise: éléments d'histoire de la médecine (Nantes 2006)

Richer, Paul, Etudes cliniques sur la grande hystérie ou hystéro-épilepsie (Paris 1885)

Shapin, Steven, A Social History of Truth: Civility and Science in Seventeenth-Century England (Chicago 1995)

- /Simon Schaffer, Leviathan et la pompe à air: Hobbes et Boyle entre science et politique (Paris 1993)

Sturken, Marita/Lisa Cartwright, Practices of Looking: An Introduction to Visual Culture (Oxford 2001)

Swain, Gladys, Le sujet de la folie: naissance de la psychiatrie (Paris 1997)

Timby, Kim, «Images en relief et images changeantes. La photographie à réseau ligné», Etudes photographiques 9 (2001)

Weiser, Martin, Medizinische Kinematographie (Dresden 1919)

Wider, Todd, "The Positive Image of the Physician in American Cinema During the 1930s", Journal of Popular Film and Television 17 (1990) 139-152

Zappalà, Annick, «La médecine médiatisée: entre la médicalisation du social et la socialisation de la science», Hermès 21 (1997) 181-190 